TECHNICAL PROGRESS REPORT

\section{EXOTIC ATOMS}

By

Raymond Kunselman

University of Wyoming

Laramie, wyoming 82071

February 1, 1992 - January 31, 1993

Prepared for the Department of Energy under contract \#DE-FG02-87ER40333

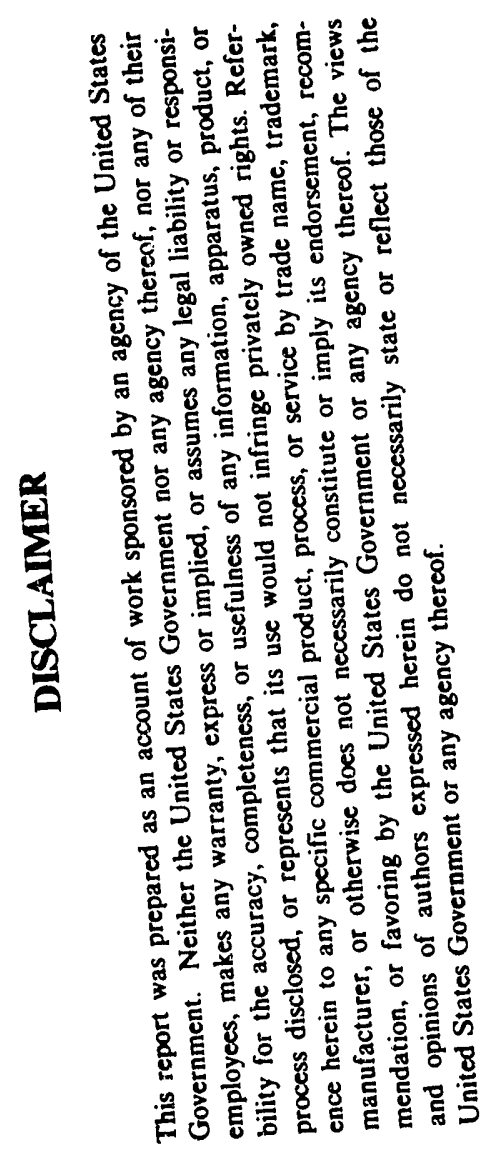

\title{
MASTER
}




\title{
EXOTIC ATOMS: Muonic Atoms into Vacuum from Solid Hydrogen
}

\begin{abstract}
The experiments use a solid hydrogen layer to form muonic hydrogen isotopes that escape into vacuum. The method relies on transfer of the muon from protium to either a deuteron or a triton. The resulting muonic deuterium or muonic tritium will not immediately thermalize because of the very low elastic cross sections, and may be emitted from the surface of the layer. Measurements which detect decay electrons, muonic x-rays, and fusion products have been used to study the processes. A target has been constructed which exploits muonic atom emission in order to learn more about the energy dependence of transfer and muon molecular formation.
\end{abstract}

\section{Introduction to reactions of muonic hydrogen}

The experimental program has averaged about two runs of beam time in recent years. It is preferable to run at the end of one period and the beginning of the next, to reduce the overhead of setting up a system of detectors which have become more and more diverse. Some runs have been shortened by TRIUMF technical problems, and this unfortunately seems to be a common situation during the start of high intensity running. Neutron detectors, a germanium detector, and a wire chamber imaging system were all operational during the latest runs [1]. Two silicon detectors were mounted in the cryostat to observe fusion products. The new two layer cryostat has been used in beam. The experiments with a single layer cryogenic target system have produced very interesting results. In order to show that fusion can be unambiguously detected in real beam environments, neutrons and protows were measured from dd fusion in a solid layer at $2.5 \mathrm{~K}$. The dd fusion neutrons were only $3 \mathrm{MeV}$ and were much more difficult to separate from capture evaporation background than the $14 \mathrm{MeV}$ neutrons from $\mathrm{dt}$ fusion. The signal to background with our apparatus in the M20B muon beam at TRIUMF was about 40 [2]. A conventional method was used to increase signal to background by demanding subsequent muon decay detection. The measurement was not trivial, and benefits from the good range width of the $30 \mathrm{MeV} / \mathrm{c}$ cloud $\mu^{-}$beam and a good delayed electron coincidence condition.

With the silicon detectors, protons from dd fusion were measured for the first time. While some problems were encountered with the operation of solid state detectors in a cryogenic hydrogen environment, the results were promising. It is planned to continue ${ }^{\star} " \ddots$. 
development of this option because alpha detection may be much more effective than neutron detection for the resonance experiment.

The germanium detector has been useful both to extend our understanding of muonic deuterium emission and to test the purity of the solid targets, which complements the $\mathrm{dd}$ fusion results. I $\mathrm{t}$ is crucial that the vacuum system is clean and that residual gases are not allowed to condense on or within the solid hydrogen. Even contaminating layers of sub-micron thickness will attenuate the muonic atom errission and contribute background. With muonic x-rays, muon transfer was observed from deuterium to a neon contaminant with concentration less than $1 \mathrm{ppm}$ (muonic $\mathrm{x}$-rays are probably the most sensitive way to measure small contaminations in deuterium) [2]. This was true even after precautions, such as palladium filtration, were taken to ensure purity. We conclude that the cryopump which now provides UHV for our target system is storing and releasing neon during target preparation. Neon, hydrogen, and helium are ubiquitous residual gases in cryopumped systems. They will be released when a load is put on the cryopump, which happens during target preparation. When target purity is important, this is not acceptable. Obviously a cryopump is not the best choice for gases which are difficult to condense. We have incorporated a turbopumping system to solve the problem. A turbopump will also relieve maintenance problems by eliminating materials which can store tritium and thus pose a safety hazard.

In February and August of 1992 we performed experiments with hydrogen and deuterium with two adjacent solid layers, to show that the purity of the layers can be independently controlled. These served as a commissioning run with the new cryostat. Another run is planned with the apparatus capable of handling tritium (but without tritium present) in order to test completely the safety system and the interaction of the new turbopump with solid targets. Only then is it planned to use tritium, in a subsequent run in 1993. The yields will be determined as they have been for $\mu^{-} \mathrm{d}$, and timing distributions will confirm the predicted low cross section for $\mu^{-} \imath$ in protium. This will be the first opportunity to measure the resonance structure.

\section{Objectives}

When a muon is stopped in hydrogen, a richly complex sequence of physical processes is initiated. The probability of a particular outcome depends upon factors such as the density $\phi$ (usually normalized to the density of liquid hydrogen), the temperature, the concentrations of the heavier hydrogen isotopes $c_{d}$ and $c_{t}$, the impurity concentration, etc.. Under a certain range of conditions, a particular sequence can lead to the emission of muonic deuterium or tritium from the surface of a solid layer into vacuum [1].

To be specific, consider the formation of muonic deuterium, $\mu^{-} \mathrm{d}$. If a muon slows in protium containing a small deuterium concentration, it will normally initially form muonic protium, $\mu^{-}$p. Transfer of the muon from the proton to a deuteron is possible because the increase in reduced mass leads to a slightly larger binding energy. This also leads to an appreciable kinetic energy (about $45 \mathrm{eV}$ ) of the $\mu^{-} \mathrm{d}$ after transfer. The average time for transfer depends on the deuterium concentration, $[2,4]$ and is of the ${ }^{\circ}$ '. 
order of $100 \mathrm{~ns}$ for $\mathrm{c}_{d}$ of order $10^{-3}$. The $\mu^{-\mathrm{d}}$ atom loses energy by elastic scattering with hydrogen, but as the energy moderates to the range of a few $\mathrm{eV}$, the cross section for scattering by a proton is drastically reduced because of the Ramsauer-Townsend (RT) mechanism. [5,6] If the deuterium concentration is not too high, $\mu^{-} \mathrm{d}$ can travel for a distance of the order of $1 \mathrm{~mm}$ before losing so much energy that the cross section is no longer within the R'T region. The muonic atom is then localized.

If the muonic atom reaches the surface of a solid hydrogen layer during its travel, it will be emitted from the layer into the adjacent region. If this region is vacuum, the muonic atom will travel unimpeded until either it reaches another material or the muon decays. Because the RT mechanism exists for muonic tritium ( $\left.u^{-} t\right)$ in protium, the same situation arises. Note that these are the only isotopic mixtures where it is expected; for example, there is no analogous RT minimum for muonic tritium in deuterium.

Experiments have been performed at TRIUMF over the past several years with the aim of understanding the factors which control emission of muonic deuterium from a solid layer. Several approaches have been taken to learn how the muonic atoms interact, using information from the time and position distributions of decay electrons, from muonic $x$-rays following transfer of the muon, and from the products of muon induced fusion. It was realized that it should be possible to extend the experiments to muonic tritium. The main motivation is to learn of the energy dependence of muon molecular formation, which is vital in the understanding of muon catalyzed fusion. A cryogenic system has been constructed to allow the use of tritium in small concentrations in solid hydrogen. It also features a second solid hydrogen layer which can be used as a target for muonic tritium emitted from the first layer, enabling certain energy measurements to be based on time of flight.

\section{Basic processes and measurements}

To understand the mechanisms which support hot muonic atom emission from solid hydrogen, it was necessary to carry out experiments under conditions of differing target thickness and deuterium concentration. A relatively simple model has been shown to demonstrate qualitatively the dependences observed. More precise comparisons will take place with a monte carlo simulation which is under development.

The emission is easily studied by reconstructing the path of muon decay electrons in order to infer the position of decay. The details of this procedure are given elsewhere, $[7]$ but the important points are as follows. A system of three wire chambers measures spatial coordinates of the electron, and scintillator counters and a sodium iodide crystal determine the time and energy of the electron respectively. The energy measurement is used simply to reject lower energy background and in some cases to improve position resolution by choosing only electrons with energy above a certain threshold; a lower energy decay electron undergoes a larger average scattering angle in material in its path. The time measurement is more important, because muons stopping in heavier elements (such as $\mathrm{Au}$ ) which make up the cryostat are quickly absorbed. This means" '•. 
that the major source of electrons later than $0.5 \mu \mathrm{s}$ after the muon arrival time is from muon decay in hydrogen. In addition, the correlation of time and position is a measure of the component of velocity perpendicular to the solid hydrogen layer. The position of decay is estimated by extrapolation of the three electron coordinates to a plane containing the beam axis. The effect of $\mu^{-} \mathrm{d}$ emission is clearly observed in histograms of the decay time for events in which the muon decays in a particular spatial region. The nost striking behaviour is where two such regions have been chosen. Rather than a purely exponential dependence, there exists a structure with a maximum at several microseconds. This is due to the emitted $\mu^{-} \mathrm{d}$ entering the chosen spatial region at a time considerably later than the arrival of the muon in the hydrogen layer. The sharper but much less intense structure at zero time shows the short lifetime of muons which stop in Au cryosiat components rather than in hydrogen. By selecting decays which occur within a particular range in time and space, the backgrounds from muons decaying normally in hydrogen or in the heavier target support materials can be virtually eliminated. For example, a typical choice is the events with a decay time between 0.5 and $5.0 \mu \mathrm{s}$. This signal has been measured as a function of density and target thickness, showing that the emission is a maximum at a concentration of about $c_{d}=10^{-3}$. The muonic atoms which are emitted originate mostly from transfer within $1 \mathrm{~mm}$ of the surface. 'This agrees with a simple model.[8] More precise comparisons with theory, which should provide some sensitivity to the energy dependence of some cross sections close to $1 \mathrm{eV}$, will be forthcoming after development and testing of a detailed monte carlo code.

Complementary information has been obtained from muonic x-rays measured by a high resolution germanium solid state detector. The eventual fate of muons is made clear by the observation of radiative transitions either after direct capture or after transfer from muonic hydrogen to heavier atoms. The two processes can be distinguished both by the different time dependences and the characteristic cascade relative intensities. This has been used to monitor possible contamination of the targets and to verify the muon stopping distribution. It has also proved possible to extract reasonably precise estimates of the rates for transfer from protium to deuterium and for muonic molecular formation in protium. The measurements are described more completely elsewhere.[1]

The solid target also provided an opportunity to measure for the first time the characteristics of muon catalyzed dd fusion at a temperature below $10 \mathrm{~K}$. A comparatively thick (several $\mathrm{mm}$ ) layer of solid pure $\mathrm{D}_{2}$ at $3 \mathrm{~K}$ was used. Of course, no emission was expected from this target, and the muons remained in the deuterium layer. Careful measurements by other groups in the liquid and gas phases at higher temperatures have existed for some time, and have been well understood in terms of hyperfine effects and muon molecular formation from thermalized $\mu^{-} \mathrm{d} \cdot[9,10]$ On that basis, no appreciable resonance formation of $\mathrm{d} \mu \mathrm{d}$ was expected. However, preliminary results show a resonance behaviour similar to that observed in warmer targets.[11] The exact interpretation of the result is not complete, and the experiments are being repeated. It is likely that the solid phase of the target modifies the processes substantially, and .. 
that the analysis which has been so successful at higher temperatures is not adequate in this case.

\section{Tritium experiment preparation}

The RT process in a solid layer can clearly be used to create energetic muonic deuterium atoms in vacuum. The typical energy of the atoms is determined by the shape of the Ramsauer-Townsend minimum in the cross section. In the case of muonic deuterium, the distribution of the longitudinal energy (i.e., the energy corresponding to the longitudinal component of velocity) has a most probable value of about $1 \mathrm{eV}$. Calculations of the RT minimum for muonic tritium in protium show a similar energy dependence, so we expect it will have a similar distribution in energy.

Recent calculations exist[12] for the rate as a function of energy in the interactions

$$
\mu \mathrm{t}+\mathrm{DX} \rightarrow[(\mathrm{d} \mu \mathrm{t}) \mathrm{xee}]
$$

where $\mathrm{x}$ is $\mathrm{p}, \mathrm{d}$, or $\mathrm{t}$ and $\mathrm{X}$ is the corresponding atomic form. The final state is a complex molecule analogous to hydrogen, where one of the "nuclei" is in fact a muonic molecule itself. It is the internal degrees of freedom of the complex molecule which lead to the resonance character of the reaction. It happens that the $\mu^{-}$t kinetic energies required to satisfy the resonance condition coincide remarkably well with the emission energy spectrum. Furthermore, the calculated rates are large enough to dominate the cross section for elastic scattering of $\mu^{-\mathrm{t}}$ by deuterons, the main mechanism for energy loss. This means that the resonant interaction may be observed by passing muonic tritium of the appropriate energy through a thin layer of HD or $\mathrm{D}_{2}$. The source of $\mu^{-\mathrm{t}}$ is the RT emitting hydrogen layer, protium with one part per thousand tritium $\left(c_{t}=10^{-3}\right)$.

A possible arrangement for the experiment is shown in ref [1]. Muons which form muonic protium and are transferred to tritons exit the emitting hydrogen layer. A second target layer is close to the emitting layer, separated by approximately $20 \mathrm{~mm}$ of vacuum. The target layer consists of up to $1 \mathrm{~mm}$ of pure protium, covered by a thin overlayer of $\mathrm{D}_{2}$ (or $\mathrm{HD}$, depending on the resonance structure we wish to measure). The thickness of the overlayer is chosen so that the probability of energy loss by the incident $\mu^{-\mathrm{t}}$ via elastic processes is small. If the $\mu^{-\mathrm{t}}$ has the appropriate resonance energy, it can interact to form the muonic molecule in the overlayer. Fusion will follow immediately to give a measurable fusion product, either a neutron or an alpha particle. Otherwise, the $\mu^{-} \mathrm{t}$ atoms may pass through to finally stop in the protium layer, where they give no signal which could be confused with a fusion event.

The energy of the incident $\mu^{-\mathrm{t}}$ is determined by its time of flight between the emitting and the target layer. The time of emission is approximately the time of muon arrival, as measured by a scintillation counter in the incident muon beam, because the 
transfer process is quite fast at the chosen tritium concentration. The time of arrival at the target layer is given by the time of detection of the fusion product, because fusion follows within nanoseconds of muon molecular formation. By far the greatest limitation on the precision of the time of flight energy determination is the unknown angle of emission of the $\mu^{-}$. A collimating device is used in the vacuum drift space to limit the angles to a range close to perpendicular with respect to the emitting surface.

\section{First results with an improved target system}

In order to proceed with the measurement described in the previous section, we have constructed a new cryogenic target system. It incorporates improvements over the initial system defined by the demands of the new experiment plus several years experience with solid hydrogen layer targets.

The features which were considered important for the new system are as follows. Two cold surfaces should be adjacent to each other, with spacing between them variable from less than $10 \mathrm{~mm}$ to $40 \mathrm{~mm}$. It must be possible to form solid layers on each surface independently, with no significant cross-contamination. The system should be capable of containing small amounts (up to $10 \mathrm{Ci}$ ) of tritium safely. Materials in which the muon beam might stop should be restricted to the hydrogen layer(s) and heavy, pure cryostat material ( $\mathrm{Au}$ or $\mathrm{Ag}$, but not both), in order to eliminate spurious muonic $\mathrm{x}$-rays and longer lived capture neutrons. The thickness of material through which the muon beam must pass before stopping in hydrogen should be minimized, in order that the beam momentum (and therefore the momentum spread) is as small as possible. Control of the temperature should be reliable at about $3 \mathrm{~K}$ with reasonable liquid helium consumption. Versatility is very important, so that different detector configurations, angular collimators, and target arrangements could be considered.

A top view of the cryostat is shown in ref [1]. It also shows an arrangement for detectors for decay electrons, muonic x-rays, and fusion products. Solid hydrogen is formed on either or both of two $50 \mu \mathrm{m}$ metal foils (currently Au, but Ag is under consideration) cooled to $3 \mathrm{~K}$. The foils are mounted by a clamping system such that their separation may be changed with only minor difficulty. A device (the diffuser) can be inserted from below; it consists of two perforated steel foils attached to a gas feed system. Hydrogen is allowed through the perforations of one foil at a slow rate and the hydrogen gas freezes, building up a layer on the foil. The diffuser can be withdrawn completely, or it can be removed such that an angular collimation device is brought betwcen the two foils. The collimator consists of hexagonal holes close packed inside a circle of size similar to the target. The transparency of the collimator to normally incident particles is greater than $90 \%$. It is made of $\mathrm{Ag}$, thus there is some motivation to make the foils also of $\mathrm{Ag}$ to reduce the types of materials in which muons may stop.

One of the first measurements with this solid target system was to prove that two dissimilar target layers could be formed without significant cross-contamination. The principle of the formation of the solid layer is that the hydrogen molecules stick almost " $\because$. 
completely at first contact with the $3 \mathrm{~K}$ surface. If they do not, it is possible that some molecules may bounce around the diffuser and stick to the other cold surface, which would make the complicated layer structure of the tritium experiment impossible. To test whether this might be a problem, an emitting layer was first deposited on one foil, and the yield of $\mu^{-} \mathrm{d}$ in the vacuum space between the targets was measured. Then a layer of pure $D_{2}$ was deposited on the opposite foil, and the measurement was repeated. If a significant amount of the $\mathrm{D}_{2}$ contaminated the surface of the first foil, the $\mu^{-} \mathrm{d}$ arriving at the surface would lose energy by scattering on deuterium and the yield would be drastically reduced. No reduction was observed, establishing that less than $1 \%$ of the deuterium was deposited on the wrong surface.

The reduction of intensity of emitted $\mu^{-} \mathrm{d}$ during passage through a thin overlayer of $\mathrm{D}_{2}$ is simply the attenuation due to the cross section. The value at $1 \mathrm{eV}$ for scattering by a deuteron is typically of order $10^{-19} \mathrm{~cm}^{2}$ larger by several orders of magnitude than for scattering by protons in the RT minimum. The effect of a deuterium overlayer on both the intensity and the longitudinal energy $E_{l}$ of the emitted at ms has been studied with the new target system. The longitudinal energy is the energy corresponding to the component of velocity along the beam direction, perpendicular to the surface of target layers, ignoring the unobserved transverse components. It is derived from the time of flight before decay, assuming the mass of $\mu^{-} \mathrm{d}$.

The intrinsic motivation for studying $E_{l}$ is twofold. First, we can hope to extract from measurements the cross section as a function of energy for a range of $\mu^{-} \mathrm{d}$ kinetic energy around $1 \mathrm{eV}$. To determine reliable values, or even to test for consistency with published calculations, [13] a comparison with detailed monte carlo calculations is clearly essential. The computer program which is being developed includes the effects of transfer, molecular formation, scattering, and emission, all of which are necessary in this case. Second, it has become clear that the properties of the emitted $\mu^{-} \mathrm{d}$ can be controlled to some extent because of the energy loss in the overlayer. Ref [1] shows longitudinal energy histograms based on muons decaying between 10 and $30 \mathrm{~mm}$ from an emitting layer, for two overlayer thicknesses as well as for no overlayer. In all cases, the histograms have been normalized and a similar histogram from a pure protium target has been subtracted. The reduction in intensity is obvious, but it is interesting to note the shift in the energy spectrum of the emitted $\mu^{-} \mathrm{d}$. Kecping in mind that the strongest resonances in the reactions of (1) are in the range $0.2-0.6 \mathrm{eV}$, it may be advantageous to use an overlayer to adjust the spectrum of emitted energies. The first experiments with muonic tritium emission will show whether such control is practical.

\section{Conclusions}

The emission of muonic deuterium from solid hydrogen is well established and understood semi-quantitatively, based on accepted cross sections and transfer rates. However, detailed comparisons with monte carlo calculations should make more precise estimations possible. The properties of muonic tritium emitted from a solid layer should. be very similar, but it is essential to make the measurements in order to examine any 
differences. Several applications exist for hot muonic hydrogen atoms in vacuum. A few of the possibilities have been pursued, but it is probable that others exist which have not yet been considered. 


\section{References}

[1] P.E. Knowles, et al., C00-2197-135.

[2] R. Jacot-Guillarmod et al., C00-2197-137.

[3] G.M. Marshall, et al.., C00-2197-138.

[4] A. Bertin, M. Bruno, A. Vitale, A. Placci, and E. Zavattini, Phys. Rev. A 7, 462 (1973).

[5] C. Chiccoli, V.I. Korobov, V.S. Melezhik, P. Pasini, L.I. Ponomarev, and J. Wozniak, INFN (Bologna) preprint INFN/BE-91/09 (1991).

[6] James S. Cohen and Michael C. Struensee, Phys. Rev. A 43, 3460 (1991).

[7] B.M. Forster et al., in Proceedings of the 5th International Conference on Muon Spin Rotation, Relaxation, and Resonance ( $\mu S R$ 90), Oxford, 1990, edited by S.F.J. Cox, G.H. Eaton, D. Herlach, and V.P. Koptev, Hyperfine Interactions 65, 1007 (1990); G.M. Marshall et al., K.R. Kendall, in Proceedings of an International Symposium on Muon Catalyzed Fusion $\mu C F-89$, Oxford, 1989, edited by J.D. Davies, (Rutherford Appleton Laboratory Report RAL-90-022).

[8] G.M. Marshall et al., in Proccedings of the Workshop on the Future of Muon Physics, Ileidelberg, 1991, (to be published in Zeitschrift für Physik C - Particles and Fields).

[9] J. Zmeskal et al., Phys. Rev. A 42, 1165 (1990).

[10] L.I. Menshikov et al., Zh. Eksp. Teor. Fiz. 92, 1173 (1987) [Sov. Phys. JETP 65, 656 (1987)].

[11] P. Kammel, MCF-92, Uppsala, Sweden, 1992.

[12] M.P. Faifman and L.I. Ponomarev, Phys. Lett. B 265, 201 (1991).

[13] V.S. Melezhik and J. Wozniak, Phys. Lett. A 116, 370 (1986). 


\section{Publications}

C00-2197-133 "Exotic Atoms," R. Kunselman, Technical Progress Report, 1992.

C00-2197-134 R. Jacot-Guillarmod, J.M. Bailey, G.A. Beer, P.E. Krowles, G.R. Mason, A. Olin, J.L. Beveridge, G.M. Marshall, J.H. Brewer, B.M. Forster, T.M. Huber, P. Kammel, J. Zmeskal, A.R. Kunselman, and C. Petitjean, Muon transfer from hot muonic hydrogen atoms to neon, presented at the international Workshop on Muon Catalyzed Fusion, $\mu \mathrm{CF}$ 92, Uppsala, June 28-July 3, 1992 (to be published in Muon Catalyzed Fusion, J.C. Baltzer AG).

C00-2197-135 P.E. Knowles, G.A. Beer, G.R. Mason, A. Olin, J.M. Bailey, J.L. Beveridge, G.M. Marshall, J.H. Brewer, B.M. Forster, T.M. Huber, R. JacotGuillarmod, L. Schellenberg, P. Kammel, J. Zmeskal, A.R. Kunselman, C.J. Martoff, and C. Petitjean, Producing $\mu \mathrm{d}$ and $\mu \mathrm{t}$ in vacuum, presented at the International Workshop on Muon Catalyzed Fusion, $\mu$ CF-92, Uppsala, June 28-July 3, 1992 (to be published in Muon Catalyzed Fusion, J.C. Baltzer AG).

C00-2197-136 G.M. Marshall, J.L. Beveridge, J.M. Bailey, G.A. Beer, P.E. Knowles, G.R. Mason, A. Olin, J.H. Brewer, B.M. Forster, T.M. Huber, B. Pippitt, R. Jacot-Guillarmod, L. Schellenberg, P. Kammel, J. Zmeskal, A.R. Kunselman, C.J. Martoff, and C. Petitjean, Experiments with energetic $\mu \mathrm{d}$ and $\mu$ t emitted from solid hydrogen, presented at the INTERNATIONAL WORKShop on Muon Catalyzed Fusion, $\mu$ CF-92, Uppsala, June 28-July 3, 1992 (to be published in Muon Catalyzed Fusion, J.C. Baltzer AG).

C00-2197-137 R. Jacot-Guillarmod, J.M. Bailey, G.A. Beer, P.E. Knowles, G.R. Mason, A. Olin, J.L. Beveridge, G.M. Marshali, J.H. Brewer, B.M. Forster, T.M. Huber, P. Kammel, J. Zmeskal, A.R. Kunselman, and C. Petitjean, Detection of hot muonic atoms emitted in vacuum using $x$-rays, presented at the Ascona Worksilop on Muonic AToms and Molecules, Ascona, April 5-9, 1992 (to be published by Birkhäuserverlag, Basel). 
C00-2197-138 G.M. Marshall, J.L. Beveridge, J.M. Bailey, G.A. Beer, P.E. Knowles, G.R. Mason, A. Olin, J.H. Brewer, B.M. Forster, T.M. Huber, B. Pippitt, R. Jacot-Guillarmod, L. Schellenberg, P. Kammel, J. Zmeskal, A.R. Kunselman, C.J. Martoff, and C. Petitjean, Hot muonic deuterium and tritium form cold targets, presented at the Ascona Worksilo? ON MUONIC Atoms And Molecules, Ascona, April 5-9, 1992 (to be published by Birkhäuserverlag, Basel).

C00-2197-139 "Exotic Atoms," R. Kunselman, Renewal Proposal Submitted to the Department of Energy, 1992. 

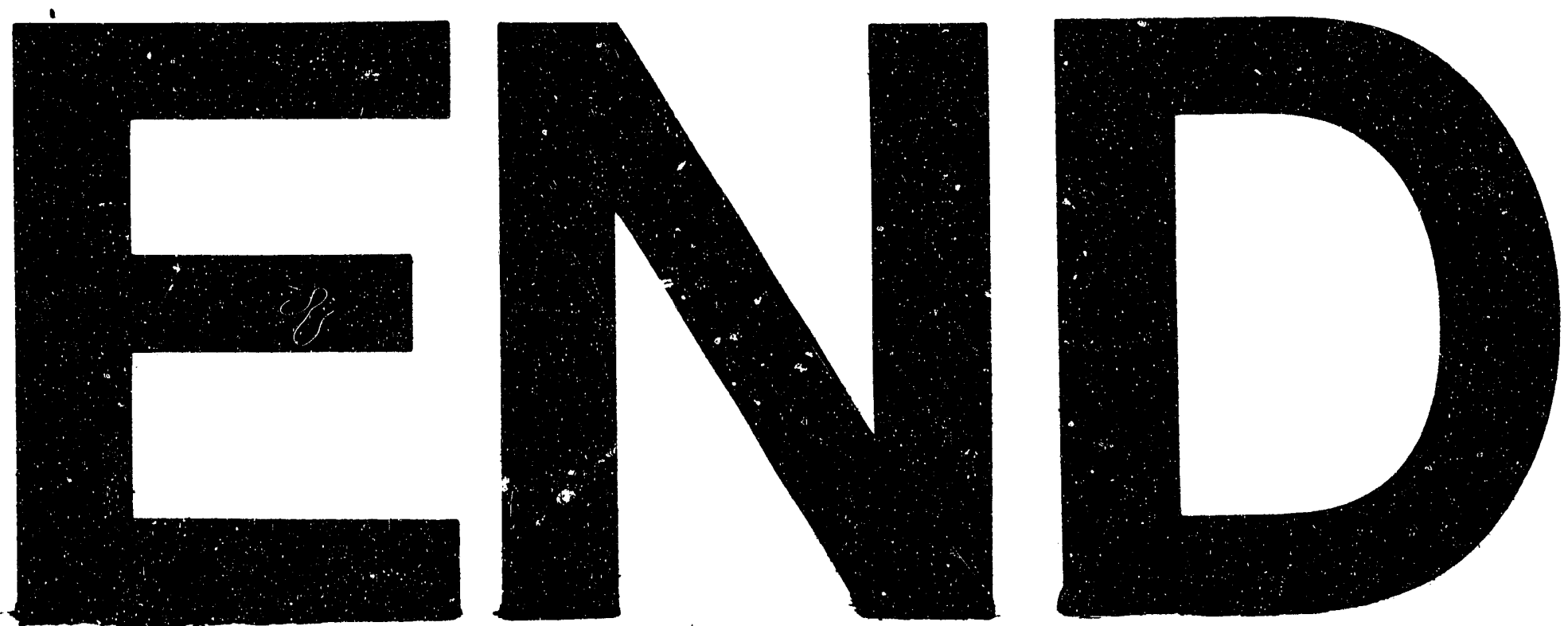

tont
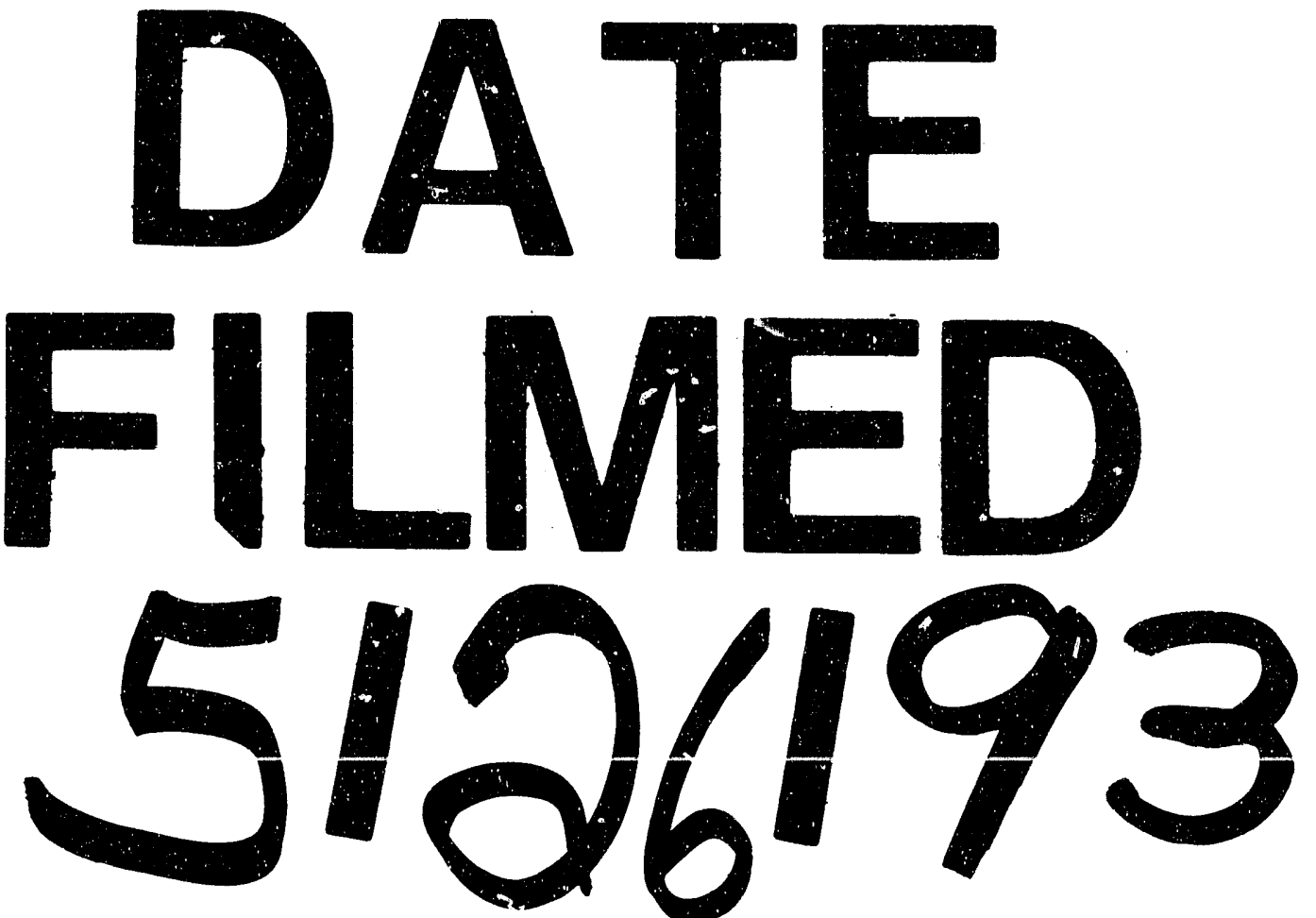
1 